\section{THE EFFECT OF INTRAUTERINE GROWTH RESTRICTION ON CIRCULATING \\ CONCENTRATIONS OF BONE COLLAGEN \\ MARKERS IN FULL-TERM PREGNANCIES}

D.D. Briana, M. Boutsikou, D. Gourgiotis,

A. Karali, A. Marmarinos, A. Georgiadis, S. Baka,

D. Hassiakos, A. Malamitsi-Puchner

Athens University Medical School, Athens, Greece

Background and aims: Intrauterine-growthrestriction (IUGR) is associated with impaired fetal skeletal development. We aimed to investigate the effect of IUGR on bone turnover in mother/infant pairs, by determining circulating bone markers of type I collagen in full-term IUGR and appropriatefor-gestational-age-(AGA) pregnancies.

Methods: Circulating markers of bone formation (carboxy-terminal propeptide of type I procollagenPICP) and resorption (cross-linked telopeptide of type I collagen-ICTP) were measured by EIA and RIA, respectively, in 40 mothers and their 20 asymmetric IUGR and 20 AGA full-term fetuses and neonates on postnatal day 1-(N1) and 4-(N4).

Results: Fetal PICP, as well as fetal and N4 ICTP concentrations were higher in the IUGR group $(p=0.036, p=0.038$ and $p<0.001$, respectively). In both groups, maternalPICP and ICTP concentrations were lower than fetal, N1 and N4 ones $(p<0.001$ in each case). In a combined group, N4 PICP concentrations were elevated compared to $\mathrm{N} 1$ ones $(p=0.012)$. Fetal ICTP concentrations were higher in females $(p=0.008)$.

Conclusions: Type I collagen synthesis and degradation are higher in IUGR fetuses/neonates. Thus, we may speculate that IUGR may affect bone mineral density and bone protein matrix in opposite directions. Higher fetal/neonatal PICP and ICTP concentrations, as compared to maternal ones, may be attributed to the high rate of skeletal growth in the former. The postnatal rapid increase in bone metabolism may account for the gradual elevation in PICP concentrations during the first days of life. Finally, the higher fetal ICTP concentrations in females may be explained by gender-related differences in bone mass.
383

\section{THE T1405N CARBAMOYL PHOSPHATE SYNTHETASE POLYMORPHISM DOES NOT AFFECT PLASMA ARGININE CONCENTRATIONS IN PRETERM INFANTS}

R.M. Moonen ${ }^{1}$, I. Reyes ${ }^{2}$, G. Cavallaro ${ }^{3}$, G. González-Luis ${ }^{2}$, J.A. Bakker ${ }^{4}$, E. Villamor ${ }^{5}$

${ }^{1}$ Paediatrics, Atrium Medical Centre Parkstad, Heerlen, The Netherlands, ${ }^{2}$ Paediatrics, Hospital Universitario Materno-Infantil de Canarias, Las

Palmas de Gran Canaria, Spain, ${ }^{3}$ Paediatrics and Neonatology, Fondazione IRCCS Ospedale Maggiore Policlinico, Mangiagalli e Regina Elena, University of Milan, Milan, Italy, ${ }^{4}$ Clinical Genetics, ${ }^{5}$ Paediatrics, Maastricht University Medical Center (MUMC+), Maastricht, The Netherlands

Background: A C-to-A nucleotide transversion (T1405N) in the gene that encodes carbamoylphosphate synthetase 1 (CPS1) has been associated with changes in plasma concentrations of L-arginine in term and near term infants but not in adults. In pretem infants homozygosity for the CPS1 Thr1405 variant (CC genotype) was associated with an increased risk of having necrotizing enterocolitis (NEC). Plasma L-arginine concentrations are decreased in preterm infants with NEC.

Aim: To examine the putative association between the CPS1 T1405N polymorphism and plasma arginine concentrations in preterm infants.

Methods: Prospective multicenter cohort study. Plasma and DNA samples were collected from 128 preterm infants (<30 weeks) between 6 and 12 hours after birth. Plasma amino acid and CPS1 T1405N polymorphism analysis were performed.

Results: Distribution of genotypes did not differ between the preterm (CC:CA:AA $=55.5 \%: 33.6 \%: 10.9 \%, n=128)$ and term infants (CC:CA:AA $=54.2 \%: 35.4 \%: 10.4 \%, n=96$ ). There was no association between the CPS1 genotype and plasma L-arginine or L-citrulline concentration, or the ornithine to citrulline ratio, which varies inversely with CPS1 activity. Also the levels of asymmetric dimethylarginine, and symmetric dimethylarginine were not significantly different among the three genotypes.

Conclusions: The present study in preterm infants did not confirm the earlier reported association between CPS1 genotype and L-arginine levels in term infants. 